\title{
COMPARING PROPERTIES OF BITUMINOUS CONCRETE MIXES PREPARED USING MORTH AND SUPERPAVE GRADATIONS
}

\author{
P. Sarika ${ }^{1}$, A K Sandra ${ }^{2}$ \\ ${ }^{1}$ Post Graduate Student, University of Texas, Arlington \\ ${ }^{2}$ Manager, GMR Infrastructure Ltd., RGI Airport, Hyderabad
}

\begin{abstract}
Hot Mix Asphalt (HMA) consists of two basic ingredients: aggregate and asphalt binder. HMA mix design is the process of determining Optimum Binder Content which satisfies the Marshall Properties. In the mix design process, selection of suitable aggregate gradation plays an important role in performing the pavement during its service life. In India, MoRTH guidelines are to be followed in selecting the aggregate gradation. Sometimes, it is difficult to achieve the gradation specified by MoRTH due to restrictions in upper and lower limits. It was observed that the gradation specified in SuperPave Mix design which is followed by majority of countries worldwide has little flexibility as compared to the MoRTH gradation. Hence, in the present study Marshall Properties were studied by following two aggregate gradations MoRTH and Superpave. The results obtained from the laboratory experiments shown that the SuperPave aggregate gradation is superior than the MORTH aggregate gradation in almost all the criterions of strength and volumetric parameters.
\end{abstract}

Keywords: Hot Mix Asphalt (HMA), MoRTH, Superpave $* * *$

\section{INTRODUCTION}

The top layers of a flexible pavement, particularly the surface course has to withstand high stress conditions and wear and tear due to traffic loads. In addition, the surface course is exposed to adverse climatic factors including temperature variations, water, etc. Therefore, properly designed high quality hot bituminous mixtures are to be laid on the surface course of flexible pavement. The high quality bituminous mixes consist of well graded hard aggregates and suitable bituminous binder of correct proportion. In the bitumen concrete mixes, 95 percent by weight is occupied by aggregate and hence, the structure formation in terms of gradation plays an important role [1,2].

The particle size distribution, or gradation, of an aggregate is one of the most influential aggregate characteristics in determining how it will perform as a pavement material. In Hot Mix Asphalt (HMA), important performance related parameters including stiffness, stability, durability, permeability, workability, fatigue resistance, frictional resistance and moisture susceptibility depends on aggregate gradation [3, 4]. In PCC, performance parameters including durability, porosity, workability, cement and water requirements, strength, and shrinkage depends on distribution of aggregate particles. Because of this, gradation is a primary concern in HMA and PCC mix design and thus most agencies specify allowable aggregate gradations for both.

Since, the gradation has a propound effect on material performance, it is necessary to select the best gradation. In general, the best gradation is nothing but the gradation which produce the maximum density. This would involve a particle arrangement where smaller particles are packed between the larger particles, which reduce the void space between particles. This creates more particle-to-particle contact, which in HMA would increase stability and reduce water infiltration. In case of HMA mixes voids space is important to occupy the volume of bitumen and to coat over the aggregate. Also, some more void space is required to readjust the bitumen when it became soft during hot climate and due to re-adjustment of particles due to secondary compaction. Considering these factors a suitable aggregate gradation is very much essential. In the present study six aggregate gradings were designed, three as per Ministry of Road Transport and Highways (MoRTH) [5] and three as per SuperPave. At various bitumen content, Marshall Mixes were prepared and tested for its properties.

\section{MATERIALS USED.}

\subsection{Asphalt Cement}

Asphalt cement of grade VG30 obtained from HPCL Visakhapatnam, AP, India, was used for the study. Table 1 shows the physical tests of the asphalt. 
Table 1: Asphalt Cement Physical Properties

\begin{tabular}{|l|l|l|l|}
\hline Test & Specifications & Result & Spec. limit \\
\hline Penetration, mm & IS-1203 & 56 & $50-70$ \\
\hline Ductility, mm & IS-1208 & 41 & Min. 40 \\
\hline Softening point & IS-1205 & 48 & Min. 47 \\
\hline Viscosity at $60^{\circ} \mathrm{C}$, Poise (min) & IS-1206 & 3250 & $2400-3600$ \\
\hline Viscosity at $135^{\circ} \mathrm{C}, \mathrm{cSt}(\mathrm{min})$ & IS-1206 (Part 2) & 365 & Min 350 \\
\hline PG Grade & AASHTO T 315 & 64 & \\
\hline
\end{tabular}

\subsection{Aggregate}

The aggregate used in this work is obtained from Medchal, Hyderabad, AP, India. The gradations of aggregate of four sizes, coarse aggregate, fine aggregate, dust and lime were used to prepare the aggregate blends to meet the aggregate gradation specifications. The following section describes the properties of the aggregate.

\subsubsection{Physical Properties of Aggregates:}

To check whether the aggregates suitable for bitumen mixes, Important physical properties such as Particle shape, strength tests, specific gravity and water absorption tests have been conducted and data is as follows.

Table 2: Particle Shape and Strength Tests

\begin{tabular}{|l|l|l|l|l|l|}
\hline Sl.No & Property & Name of the Test & Test Result & $\begin{array}{l}\text { Specification } \\
\text { Limit } \\
\text { (MORT\&H) }\end{array}$ & Test Method \\
\hline 1 & Particle Shape & $\begin{array}{l}\text { Combined Flakiness \& } \\
\text { Elongation Indices of Aggregate }\end{array}$ & $23.3 \%$ & Max. 35\% & IS:2386 Part-1 \\
\hline 2 & Strength & Aggregate Impact Value & $21.5 \%$ & Max. 24\% & IS:2386 Part-4 \\
\hline
\end{tabular}

Table 3: Specific Gravity and water absorption of Aggregates

\begin{tabular}{|l|l|l|l|}
\hline Size of Agg. & $\begin{array}{l}\text { Bulk Sp.Gr } \\
\text { (Oven Dry) }\end{array}$ & ApparentSp.Gr & Water Absorption \\
\hline $26.0 \mathrm{~mm}-19.0 \mathrm{~mm}$ & 2.652 & 2.666 & 0.19 \\
\hline $19.0 \mathrm{~mm}-13.2 \mathrm{~mm}$ & 2.650 & 2.665 & 0.21 \\
\hline $13.2 \mathrm{~mm}-9.5 \mathrm{~mm}$ & 2.648 & 2.664 & 0.23 \\
\hline $9.5 \mathrm{~mm}-4.75 \mathrm{~mm}$ & 2.645 & 2.662 & 0.25 \\
\hline $4.75 \mathrm{~mm}-2.36 \mathrm{~mm}$ & 2.644 & 2.665 & 0.30 \\
\hline $2.36 \mathrm{~mm}-1.18 \mathrm{~mm}$ & 2.641 & 2.672 & 0.44 \\
\hline $1.18 \mathrm{~mm}-0.600 \mathrm{~mm}$ & 2.640 & 2.683 & 0.60 \\
\hline $0.600 \mathrm{~mm}-0.300 \mathrm{~mm}$ & 2.638 & 2.700 & 0.87 \\
\hline $0.300 \mathrm{~mm}-0.150 \mathrm{~mm}$ & 2.638 & 2.729 & 1.26 \\
\hline $0.150 \mathrm{~mm}-0.075 \mathrm{~mm}$ & 2.636 & 2.757 & 1.67 \\
\hline $0.075 \mathrm{~mm}$ passing material & 2.630 & 2.857 & 3.01 \\
\hline Filler (Lime) & 2.420 & 2.420 & \\
\hline & & & 0.51 \\
\hline
\end{tabular}

Note: As per MORT\&H specifications water absorption of combined Coarse $\&$ fine aggregates should not be more than $2 \%$ thus comply the specification limits.

Specific Gravity of lime: 2.420

Specific gravity of Bitumen: 1.052

\subsubsection{Individual Aggregate Gradations}

Individual aggregategradations trialswere conducted forthree sizes of aggregates i.e. $20 \mathrm{~mm}, 10 \mathrm{~mm}, 4.75 \mathrm{~mm}$ down and filler (Hydrated lime) and the results are summarized in the following Table 4. 
Table 4: Individual aggregate Gradation

\begin{tabular}{|l|l|l|l|l|}
\hline Sieve size & $\begin{array}{l}\mathbf{2 0} \mathbf{~ m m} \mathbf{\%} \\
\text { passing }\end{array}$ & $\begin{array}{l}\mathbf{1 0} \mathbf{~ m m ~ \%} \\
\text { passing }\end{array}$ & $\begin{array}{l}\mathbf{4 . 7 5} \mathbf{~ m m ~ d o w n ~} \\
\text { \% passing }\end{array}$ & $\begin{array}{l}\text { Filler } \\
\text { Passing }\end{array}$ \\
\hline 26.5 & 100.0 & 100.0 & 100.0 & 100.0 \\
\hline $19.0 \mathrm{~mm}$ & 56.4 & 100 & 100 & 100 \\
\hline $13.2 \mathrm{~mm}$ & 22.5 & 100 & 100 & 100 \\
\hline $9.5 \mathrm{~mm}$ & 3.5 & 72.6 & 100 & 100 \\
\hline $4.75 \mathrm{~mm}$ & & 8.6 & 99.9 & 100 \\
\hline $2.36 \mathrm{~mm}$ & & & 84.8 & 100 \\
\hline $1.18 \mathrm{~mm}$ & & & 66.5 & 100 \\
\hline $600 \mathrm{micron}$ & & & 44.8 & 100 \\
\hline $300 \mathrm{micron}$ & & & 29.8 & 100 \\
\hline 150 micron & & & 16.6 & 100 \\
\hline 75 micron & & & 8.2 & 98.4 \\
\hline
\end{tabular}

\section{DESIGNED AGGREGATE GRADATION AS}

PER MORTH AND SUPERPAVE

\section{SPECIFICATIONS}

The main objective of this work is to compare the Marshall Properties of various aggregate gradations designed as per MoRTH and SuperPave specifications. Hence, 6 different aggregate gradations 3 from MoRTH (fine, medium and course) gradations and 3 from SuperPave were selected. The blending of aggregate is carried out to produce three aggregate gradations goes within the MORTH and
SuperPave upper and lower limits. The first gradation trial goes near to the upper limit (fine), the second gradation trial goes as a middle gradation between upper and lower limits (medium), and third gradation trial goes near to the lower limit (course). MoRTH and SuperPave gradations are depicted in Figures 1 to 2 . In the Figure 1 of MoRTH gradation, MU denotes the fine gradation, MM denotes Intermediate Gradation and ML denotes courser gradation. Similarly, for SuperPave gradation M is replaced with the letter $\mathrm{S}$ and the designation is same.

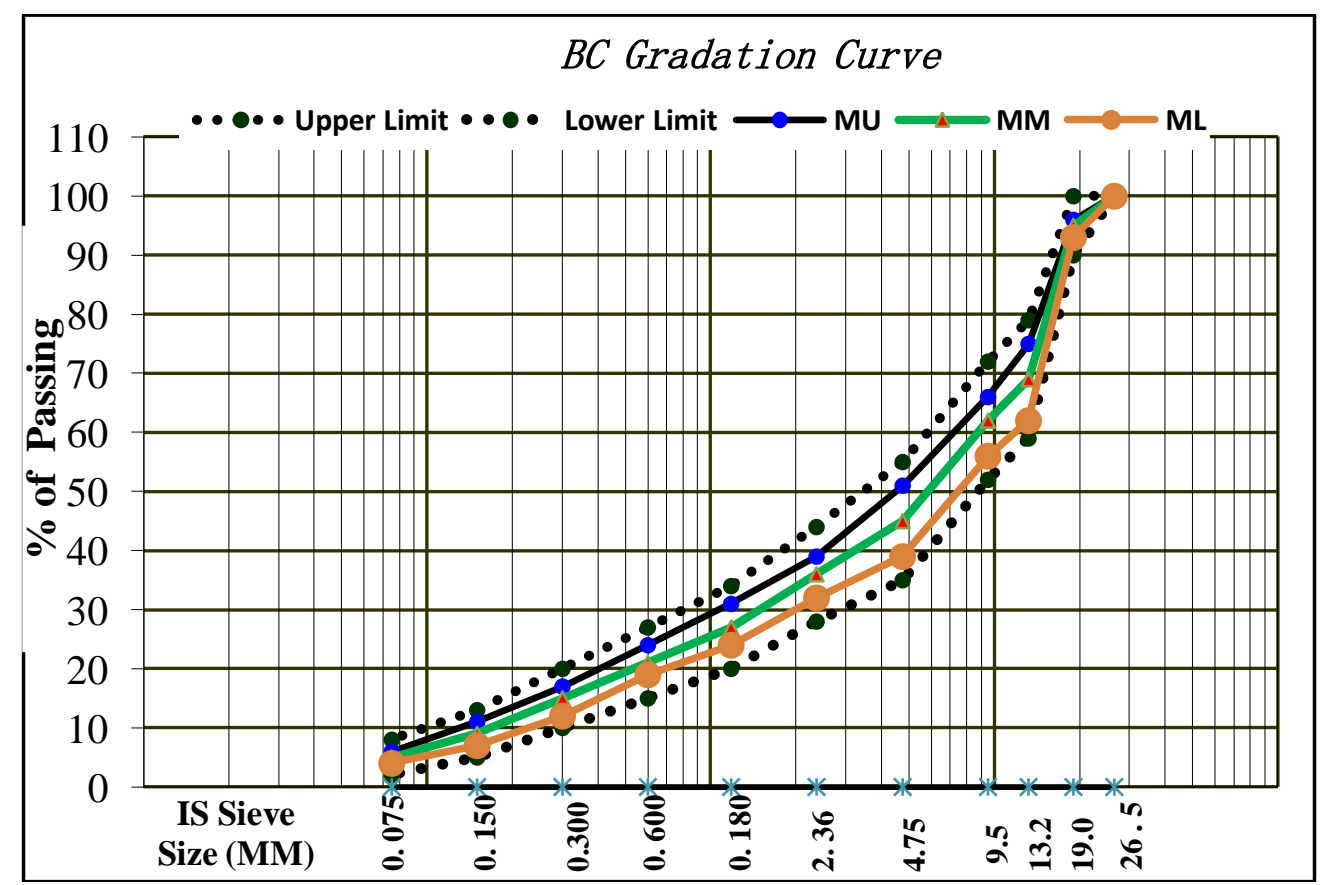

Fig 1: Aggregate Gradation as per MoRTH Specifications 


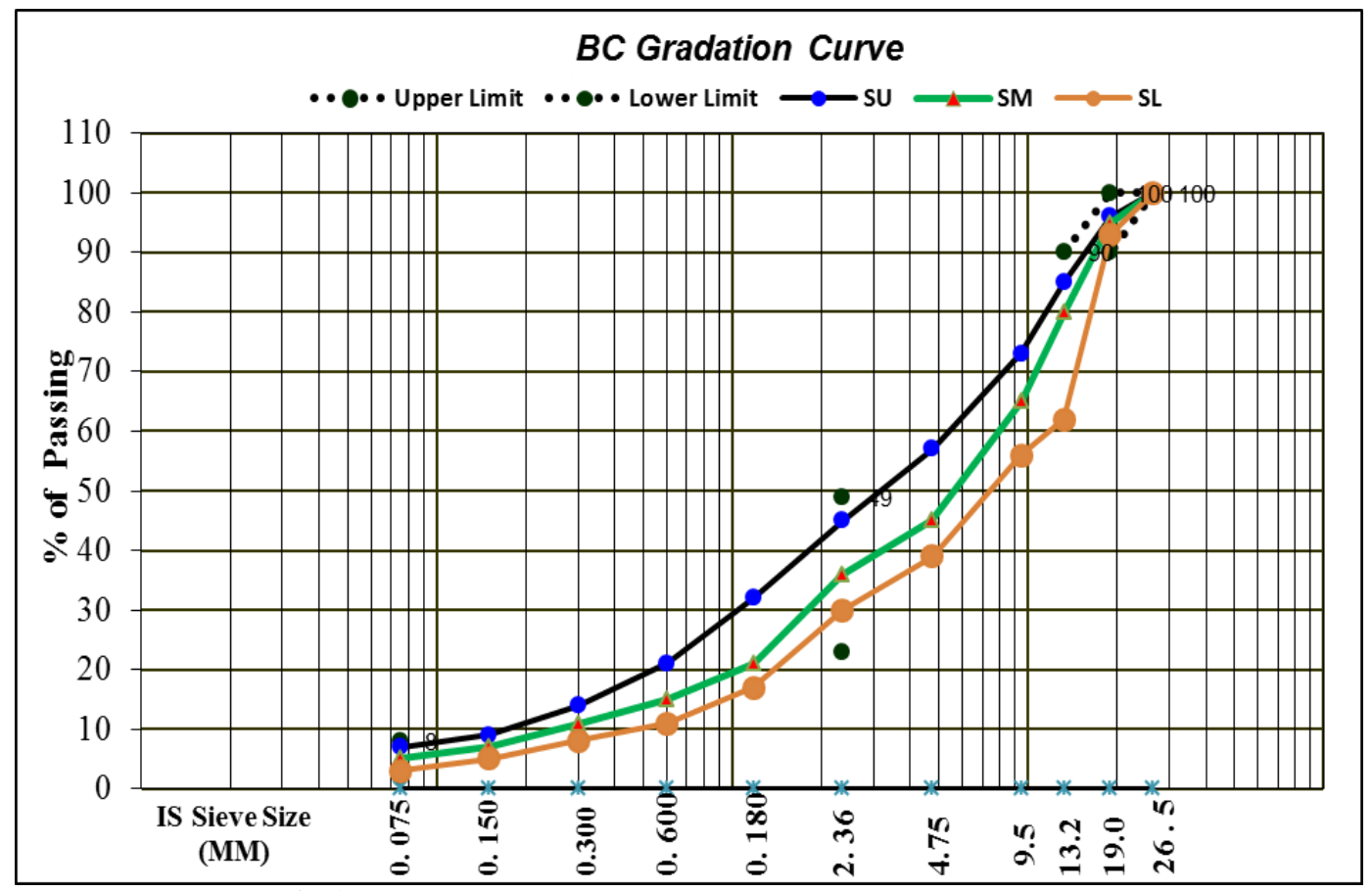

Fig 2: Aggregate Gradation as per SuperPave Specifications

\section{PREPARATION OF MARSHALL SPECIMENS:}

A set of 3 no's Marshall Specimens were casted for each aggregate gradation (total 6 gradations) by varying the bitumen content from $4.0 \%$ to $6.0 \%$ with the increment of $0.5 \%$. Total 90 Marshall specimens were casted to study the properties of the mixes. Care has been taken during preparation of Marshall specimens in terms of adding bitumen and maintaining mixing and compaction temperatures. Prepared marshal specimens were demoulded from the Marshall mould and labeled properly for future reference.

Marshall Specimen's bulk density, theoretical maximum density and volumetric properties were computed as per Asphalt Institute Manual Series - 2 (MS-2) and then specimens were tested at $60^{\circ} \mathrm{C}$ to find the Marshall Stability and flow. As per the MoRTH specifications, the Optimum Bitumen Content (OBC) is the percentage of bitumen corresponding to $4 \%$ of Air Voids. Accordingly, OBC for all the six grading were determined and reported.

\section{RESULTS AND DISCUSSIONS}

Marshall parameters Stability, Voids in Mineral Aggregates (VMA), Bulk Density, Air Voids, Flow and Voids Filled Bitumen (VFB) for all six aggregates grading were pictorially shown in the Figures 3 to 8 .

\subsection{Stability}

It is clearly observed that the Superpave aggregate gradation is superior to MoRTH gradation. The minimum stability accepted by the MoRTH specification is $9 \mathrm{kN}$. The stability values for MoRTH and SuperPave gradations are much higher than that of minimum value prescribed in guidelines. As shown in the Figure 3, the SuperPave lower gradation trial showed a more stability values as compared to the other gradations. Within the three MoRTH gradations also, lower gradations stability is more as compared to the middle and upper gradation trials. The lower gradation trial is considered the coarser gradation with respect to the middle and the upper one, which is the main reason for its strength and it is providing better aggregate internal friction with an optimum air voids. Even, in case of Superpave gradation, lower gradation trial shown its superiority on the middle and the upper gradation trails. 


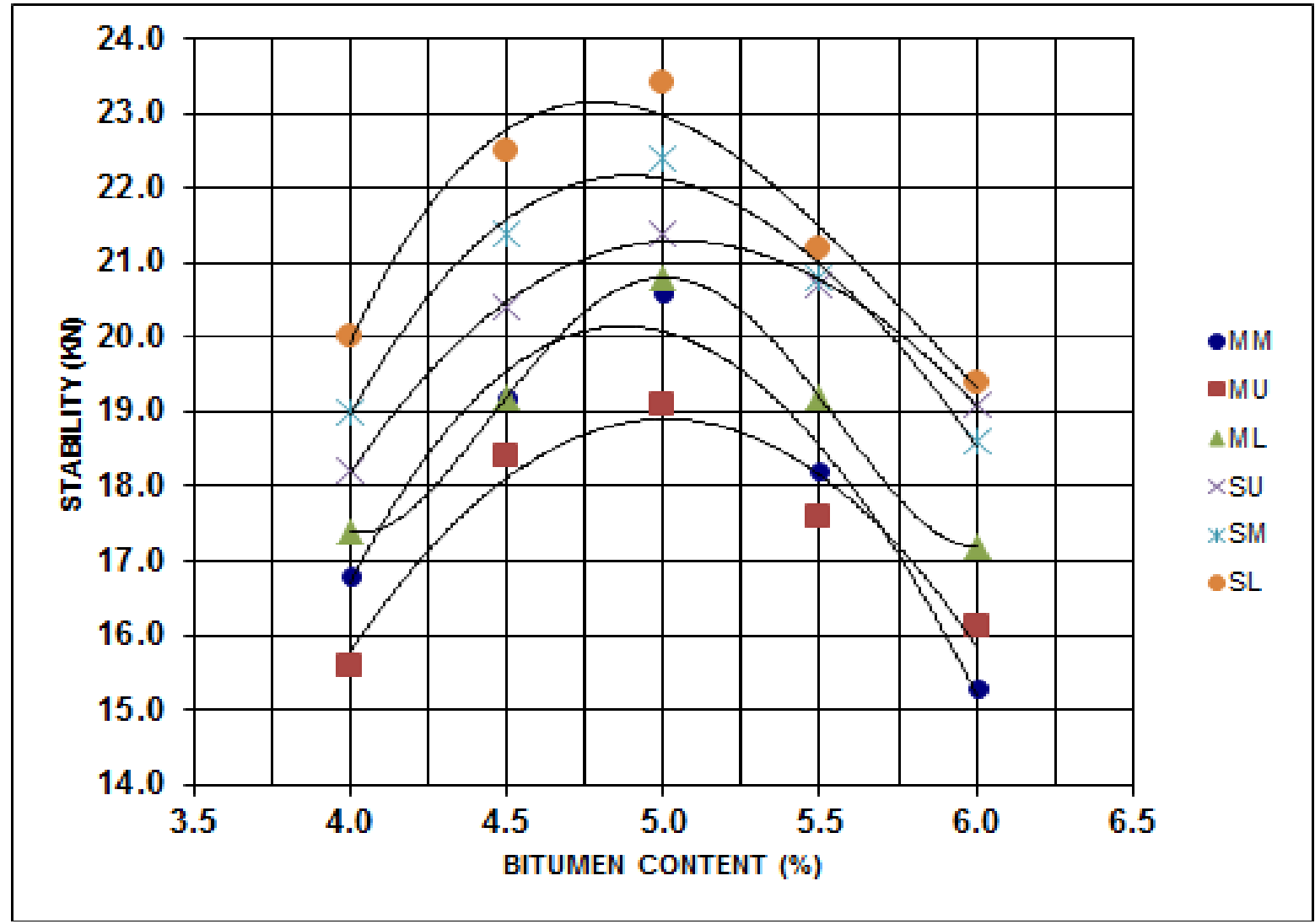

Fig 3: Stability vs Bitumen Content values for MoRTH and SuperPave Gradation

\subsection{Optimum Binder Content (OBC) from Airvoids}

As per the MoRTH specifications, OBC should be read as the bitumen content corresponding to $4 \%$ of Airvoids. Figure 4 shows that for $4 \%$ Airvoids, bitumen content various from 5\% to $5.25 \%$ for various grading. OBC of SuperPave lower grading is $5.0 \%$ and OBC of MoRTH medium grading is $5.25 \%$. Since, SuperPave lower grading is comparatively coarser and naturally, requires less bitumen content as compared to other grading.

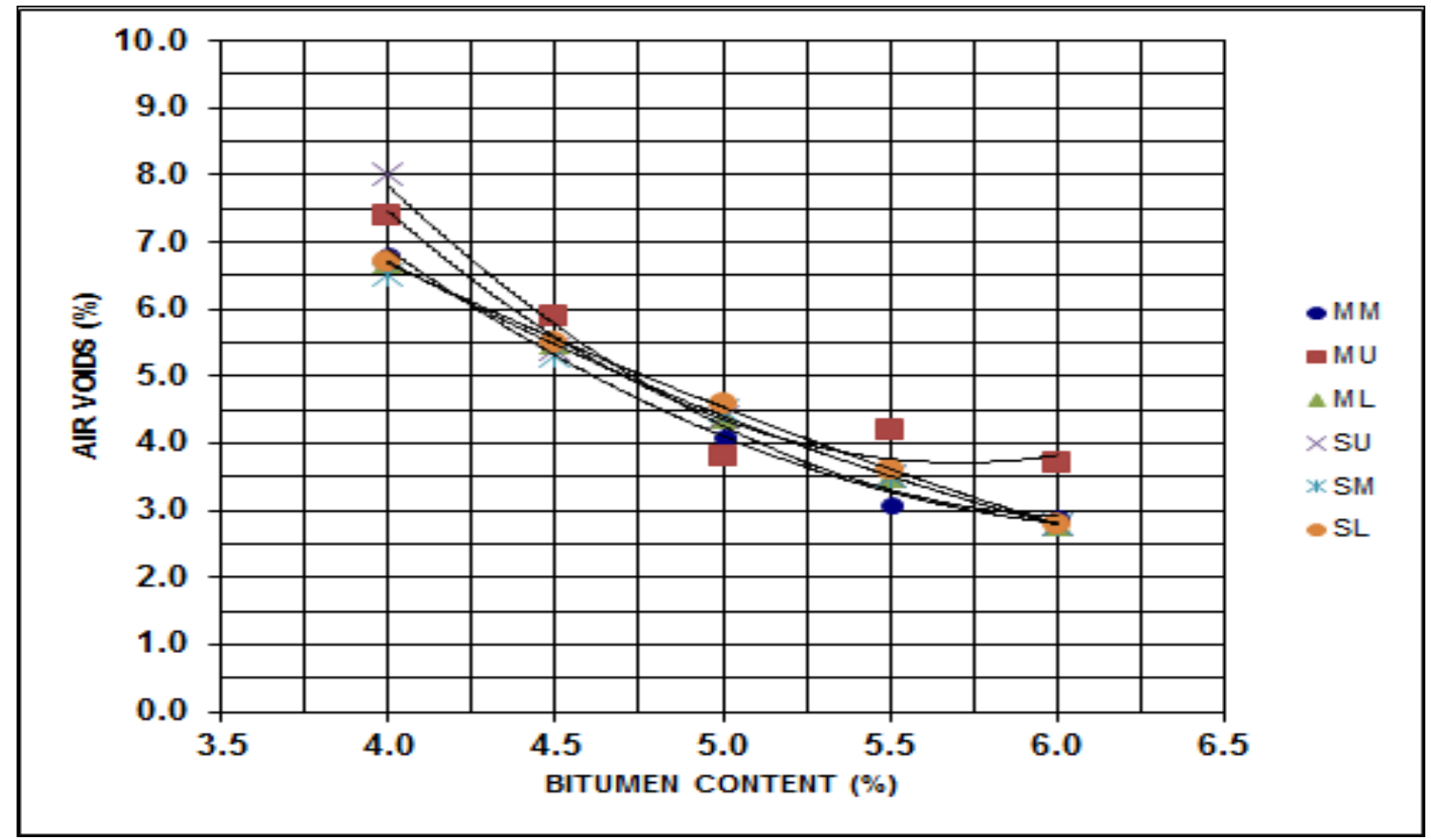

Fig 4: Airvoids vs Bitumen Content values for MoRTH and SuperPave Gradation 


\subsection{Flow Values}

MoRTH specifications suggest to have flow values in the range of 2 to $4 \mathrm{~mm}$ for the mix prepared at OBC. In this case for all the gradations, the flow values are within the limits at OBC. However, SuperPave gradations shown higher flow values as compared to the MoRTH gradations and it may be due to coarser aggregates (Figure 5).

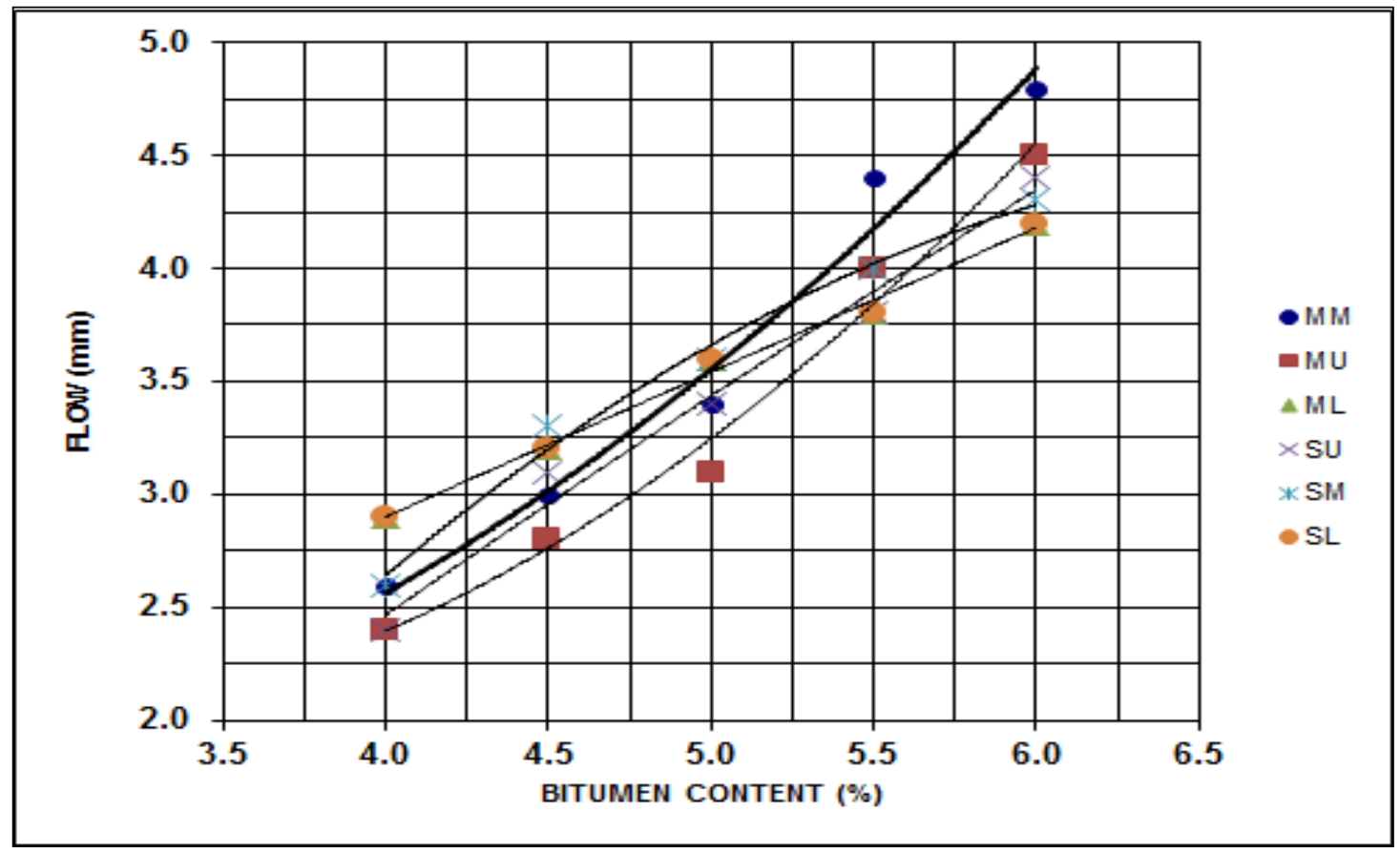

Fig 5: Flow vs Bitumen Content values for MoRTH and SuperPave Gradation

\subsection{Unit Weight}

SuperPave gradations shows higher unit weights as compared to MoRTH gradations, it is due to presence of higher coarser material. Generally, coarser particles has higher specific gravity due to less volume for the same weight as compared to the finer material and same is reflected in the unit weights also (Figue 6).

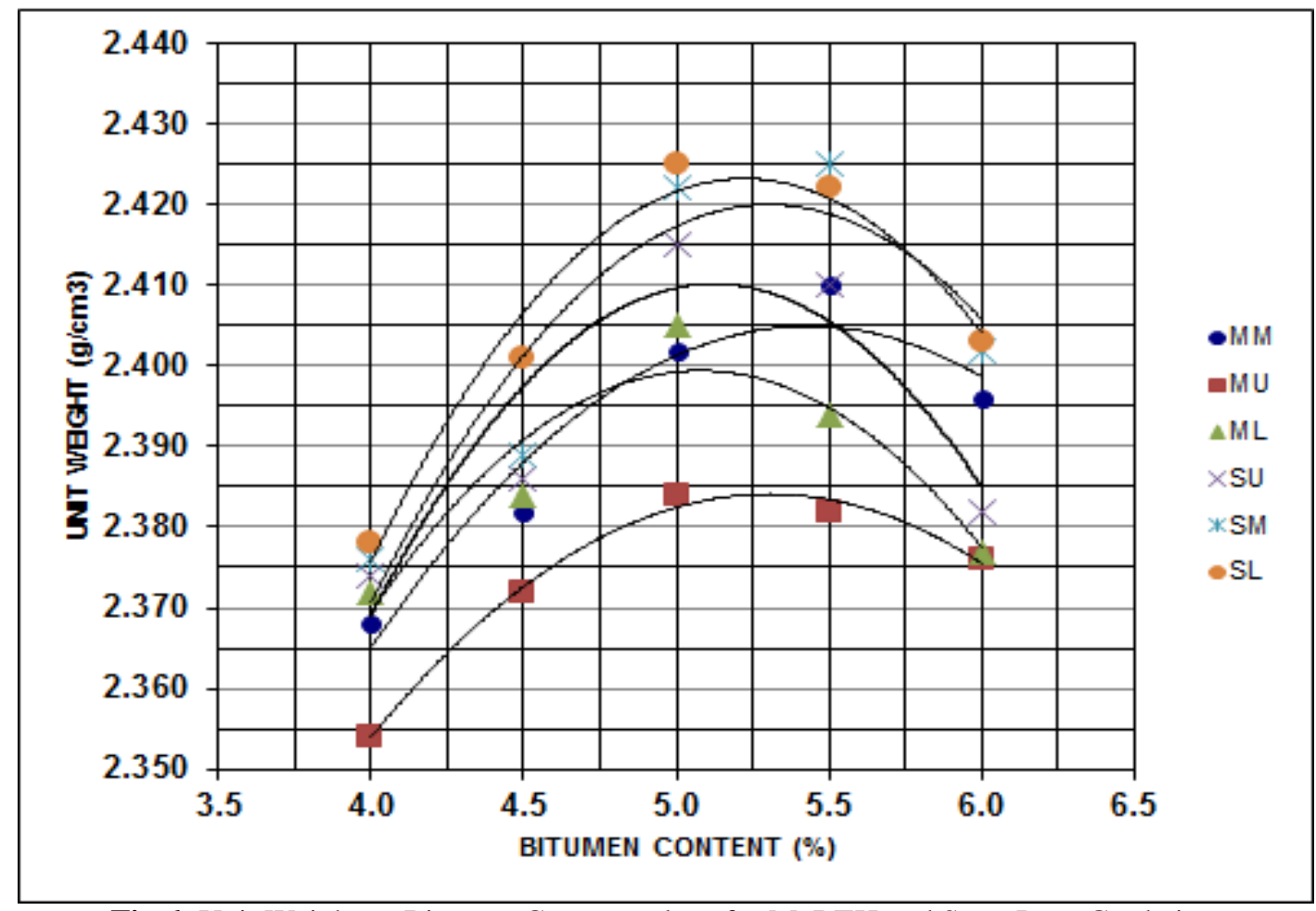

Fig 6: Unit Weight vs Bitumen Content values for MoRTH and SuperPave Gradation 


\subsection{Voids in Mineral Aggregates (VMA)}

It has been observed that all the gradation trails produced a VMA values is higher than the specifications. However, MoRTH gradations produced high VMA values as compared to SuperPave gradations. This due to finer particles has more voids as compared to the coarser particles (Figure 7).

\subsection{Voids Filler by Bitumen (VFB)}

As per the MoRTH specifications, VFB values should be in between 65 to $75 \%$. For all the aggregate gradations VFB values are within the prescribed limits at OBC (Figure 8). MoRTH upper grading looks dry as compared to other gradings due to presence of more particles whereas SuperPave lower gradation looks wet due to coarser.

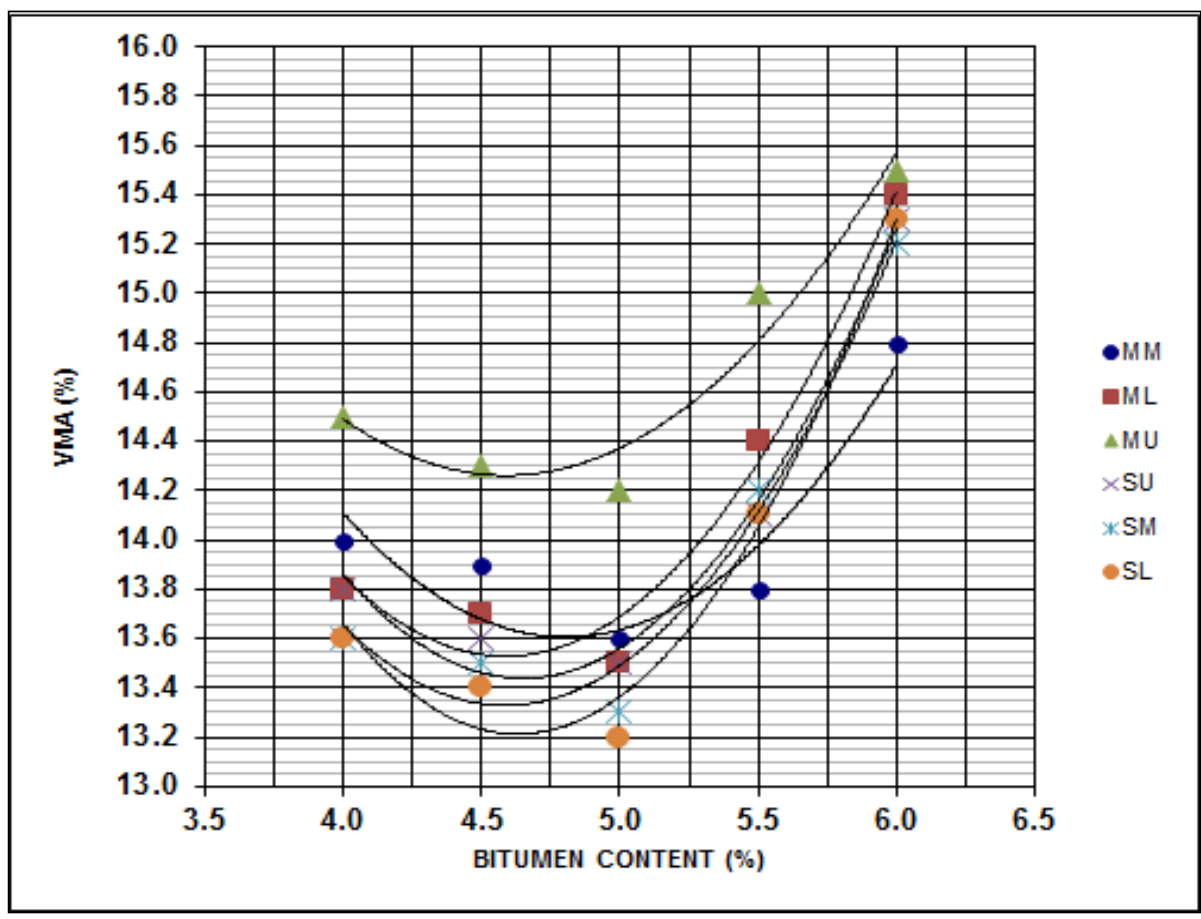

Fig 7: Unit Weight vs Bitumen Content values for MoRTH and SuperPave Gradation

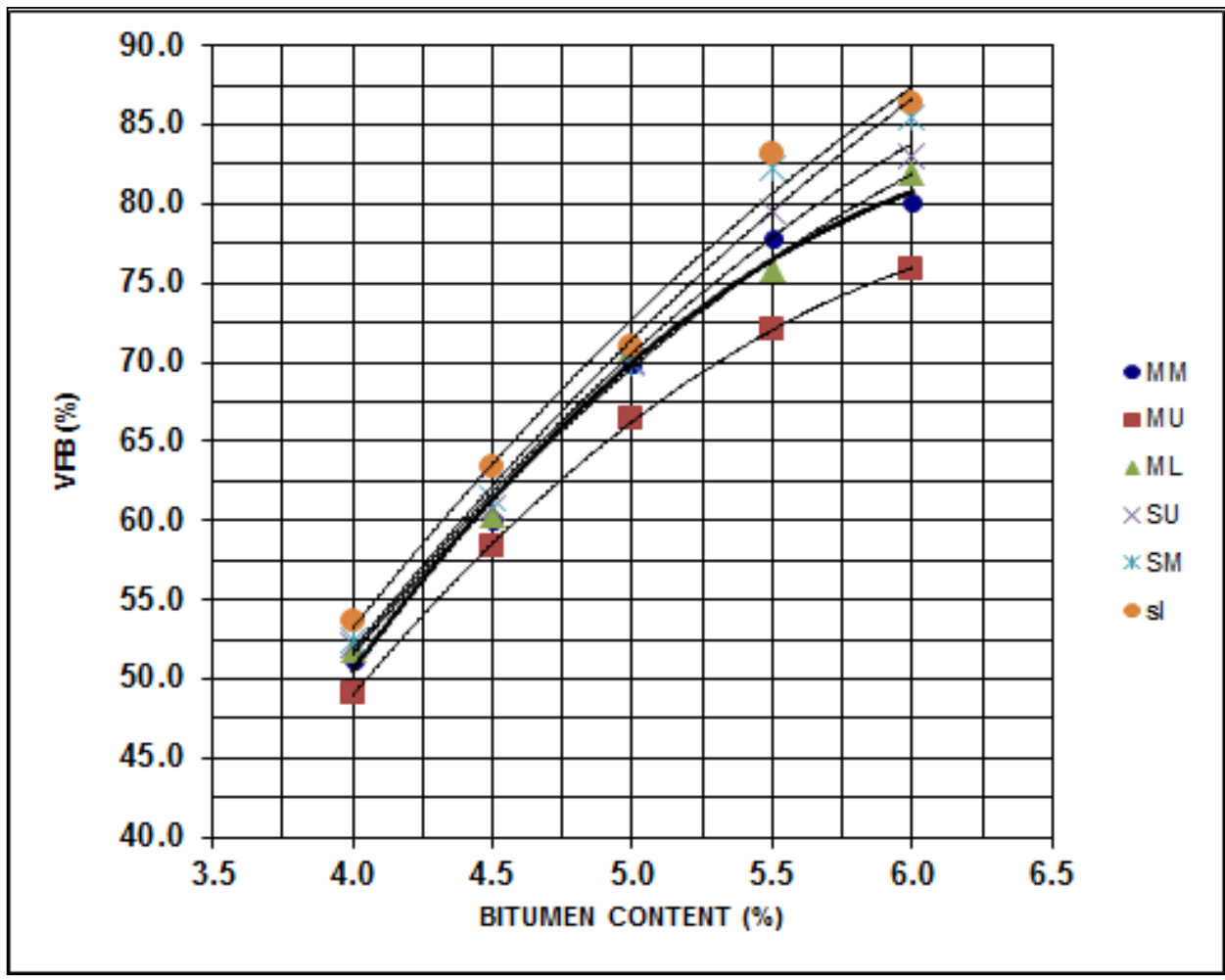

Fig 8: Unit Weight vs Bitumen Content values for MoRTH and SuperPave Gradation 


\section{CONCLUSION}

Based on the experimental analysis of MORTH and Superpave gradation data, the following conclusions were made:

- The Superpave aggregate gradation found to be superior to the MORTH aggregate gradation in Marshall Properties.

- MORTH lower gradation trial present the best results compared to the upper and middle gradations trials.

- The Superpave lower Gradation trial is the best gradation for which it shows the best results in all the Marshall criteria.

- The Superpave gradation can be considered as more economical than the MORTH gradation due less binder content consumption and more stability.

\section{REFERENCES}

[1] C.E.G.Justo. et al 2009; "Highway Materials \& Pavement Testing" Nem chand \& Bros Roorkee, India, 5th revised.

[2] National Coorperative Highway Research Program NCHRP, 2011; "A Manual for Design of Hot-Mix Asphalt with Commentary", Transportation Research Board, National Academy of Sciences. , Report 673.

[3] Roberts, F.L.; Kandhal, P.S.; Brown, E.R.; Lee, D.Y. and Kennedy, T.W. (1991); " Hot Mix Asphalt Materials, Mixture Design, and Construction",. National Asphalt Pavement Association Education Foundation. Lanham.P.no. 168:176

[4] American Association of State Highway and Transportation Officials (AASHTO). (2000c). AASHTO Provisional Standards, April 2000 Edition. American Association of State Highway and Transportation Officials. Washington, D.C.

[5] Minstry of Roads Transport and Highways (MoRTH) 2013, Specifications for Road and Bridges works, Fifth Revision, Indian Road Congress, New Delhi. 